\title{
Front Matter: Volume 10290
}

, "Front Matter: Volume 10290," Proc. SPIE 10290, Sol-Gel and Polymer Photonic Devices: A Critical Review, 1029001 (29 July 1997); doi:

$10.1117 / 12.2284585$

SPIE Event: Optical Science, Engineering and Instrumentation '97, 1997, San Diego, CA, United States 


\section{Contents}

ix Conference Committee

\section{SOL-GEL I}

3 Molecular design of oxide precursor for advanced materials [CR68-01]

L. G. Hubert-Pfalzgraf, Univ. de Nice-Sophia-Antipolis (France)

25 Sol-gel nonlinear materials based on the incorporation of nanosize crystals and fullerene derivatives [CR68-02]

M. Guglielmi, Univ. di Padova (Italy)

54 Transparent polymer/silica hybrid gels [CR68-03]

L. C. Klein, Rutgers Univ.; C. L. Beaudry, Komatsu Silicon America;

S. Yamazaki, Central Glass Co., Ltd. (Japan); A. B. Wojcik, Spec Tran Corp.

64 Optical chemical sensors based on sol-gel-derived films [CR68-04]

B. D. MacCraith, Dublin City Univ. (Ireland)

90 Polymer and ormosil materials for optical data storage [CR68-05]

F. Tang, F. Gan, Shanghai Institute of Optics and Fine Mechanics (China)

\section{SOL-GEL II}

119 Sol-gel fabrication for optical communication components: prospects and progress [CR68-06]

E. M. Yeatman, Imperial College (UK)

143 Potential of organic-inorganic hybrid materials derived by sol-gel for photonic applications [CR68-07]

A. B. Seddon, Univ. of Sheffield (UK)

172 Sol-gel technologies in thin film fabrication for integrated optics lasers and amplifiers [CR68-08]

R. M. Almeida, H. C. Vasconcelos, Instituto Superior Técnico (Portugal)

192 Chances and limitations of sol-gel-derived metal oxide and chalcogenide nanocomposites for optic and photonic application [CR68-09]

H. K. Schmidt, Institut für Neue Materialien gem. GmbH (FRG)

\section{POLYMER I}

207 Next-generation polymeric photonic devices [CR68-20]

L. A. Eldada, L. W. Shacklette, R. A. Norwood, J. T. Yardley, AlliedSignal Inc.

Sol-Gel and Polymer Photonic Devices: A Critical Review, edited by Mark P. Andrews,

S. Iraj Najafi, Proc. of SPIE Vol. 10290 (Vol. CR68), 1029001 - (c) (1997) 2017 SPIE

CCC code: $0277-786 \mathrm{X} / 17 / \$ 18 \cdot$ doi: $10.1117 / 12.2284585$

$\checkmark$

Proc. of SPIE Vol. $102901029001-1$ 
228 Polyimide-based waveguides for guided-wave multi-Gbit/sec MCM optoelectronic interconnects [CR68-21]

R. T. Chen, L. Wu, F. Li, Univ. of Texas atAustin; S. Tang, Radiant Research, Inc.; M. Dubinovsky, J. M. Qi, C. L. Schow, J. C. Campbell, Univ. of Texas

at Austin; R. W. Wickman, B. Picor, Cray Research, Inc.; M. K. Hibbs-Brenner, J. P. G. Bristow, Honeywell Technology Ctr.; Y. S. Liu, GE Corporate Research and Development Ctr.; S. Rattan, C. Noddings, Microelectronics and Computer Technology Corp.

\section{SOL-GEL III}

253 Passive and active sol-gel materials and devices [CR68-10]

M. P. Andrews, McGill Univ. (Canada); S. Najafi, Ecole Polytechnique de Montréal (Canada)

286 New developments in integrated optics using the sol-gel process [CR68-11] P. Coudray, Y. Moreau, P. Etienne, J. Porque, Univ. de Montpellier II (France)

\section{POLYMER II}

307 Designing electro-optical polymer films: optical loss vs EO activity vs stability [CR68-13]

G. A. Lindsay, Naval Air Warfare Ctr.

313 Role of intermolecular interactions in fabricating hardened electro-optic materials [CR68-14]

L. R. Dalton, A. W. Harper, J. Chen, S. Sun, S. Mao, S. Garner, A. Chen, W. H. Steier, Univ. of Southern California

322 Poling and characterization of photonic waveguide devices for efficient second-harmonic generation [CR68-19]

M. L. Jäger, G. I. Stegeman, CREOL/Univ. of Central Florida; S. Bauer, S. Bauer-Gogonea, W. Brinker, S. Yilmaz, W. Wirges,

Heinrich-Hertz-Institut (FRG)

\section{POLYMER III}

343 Current status and future of photorefractive polymers for photonic applications [CR68-15]

B. Kippelen, N. Peyghambarian, Optical Sciences Ctr./Univ. of Arizona

374 Unification of azo-polymer systems by photo-induced molecular movement [CR68-16]

Z. Sekkat, Univ. of California/Davis and Max-Planck-Institut für Polymerforschung (FRG); A. Knoesen, Univ. of California/Davis; W. Knoll, Max-Planck-Institut für Polymerforschung (FRG) and RIKEN-The Institute of Physical and Chemical Research (Japan); R. D. Miller, IBM Almaden Research Ctr. 
399 Cross-linked polyimides for integrated optics [CR68-17]

K. D. Singer, Case Western Reserve Univ.; T. C. Kowalczyk, H. D. Nguyen,

NASA Lewis Research Ctr.; A. J. Beuhler, D. A. Wargowski, Amoco

Chemical Co.

422 Polymer optical fibers for photonics [CR68-18]

M. G. Kuzyk, Washington State Univ.

vii

Proc. of SPIE Vol. $102901029001-3$

Downloaded From: https://www.spiedigitallibrary.org/conference-proceedings-of-spie on 26 Apr 2023 Terms of Use: https://www.spiedigitallibrary.org/terms-of-use 
Proc. of SPIE Vol. $102901029001-4$ Downloaded From: https://www.spiedigitallibrary.org/conference-proceedings-of-spie on 26 Apr 2023
Terms of Use: https://www.spiedigitallibrary.org/terms-of-use 


\title{
Conference Committee
}

\author{
Conference Chairs \\ Mark P. Andrews, McGill University (Canada) \\ S. Iraj Najafi, Ecole Polytechnique Montréal (Canada) \\ Session Chairs \\ 1 Sol-Gel I \\ S. Iraj Najafi, Ecole Polytechnique de Montréal (Canada)) \\ 2 Sol-Gel II \\ Mark P. Andrews, McGill University (Canada) \\ 3 Polymer I \\ S. Iraj Najafi, Ecole Polytechnique de Montréal (Canada) \\ 4 Sol-Gel III \\ S. Iraj Najafi, Ecole Polytechnique de Montréal (Canada) \\ 5 Polymer II \\ Mark P. Andrews, McGill University (Canada) \\ 6 Polymer III \\ Mark P. Andrews, McGill University (Canada)
}

ix

Proc. of SPIE Vol. $102901029001-5$

Downloaded From: https://www.spiedigitallibrary.org/conference-proceedings-of-spie on 26 Apr 2023 Terms of Use: https://www.spiedigitallibrary.org/terms-of-use 